\title{
LA MIRADA COMO CONSTRUCCIÓN CULTURAL. EL CASO DE MINA MURRAY EN DRÁCULA (1897)
}

\author{
Alejandro Lillo \\ Universitat de València
}

\begin{abstract}
Resumen: El hecho de mirar es un acto cultural que se modifica con el paso del tiempo. En las páginas que siguen se estudiará la forma de percibir la realidad de Mina Murray, protagonista de Drácula (Bram Stoker, 1897). Analizando su primer encuentro con Drácula, me esforzaré por mostrar algunos de los mecanismos sociales que actúan sobre Mina y que le permiten oponerse a él. Esas fuerzas sociales están básicamente relacionadas con el bagaje cultural de Mina y sus propios intereses, pero también con el modo de pensar imperialista, con el liberalismo de las clases medias en ascenso y con la sociedad de control, encarnada en el Panóptico, que ya empieza a consolidarse por aquellos tiempos. A través del análisis de determinados aspectos de su mirada (no todos), el presente artículo tratará de comprender mejor las distintas ideologías y fuerzas sociales que actuaban sobre los individuos durante la segunda mitad del siglo XIX.
\end{abstract}

Palabras clave: Drácula, Panóptico, liberalismo, imperialismo, Mina Murray.

The look as cultural construction. The case of Mina Murray in Drácula (1897)

Abstract: The fact of looking is a cultural act that changes over time. In the following pages the way of perceiving reality will be analysed Mina Murray, protagonist of Dracula (Bram Stoker, 1897). Analyzing his first encounter with Dracula, I will strive to show some of the social mechanisms that act on Mina and that allow him to oppose him. These social forces are basically related to Mina's cultural background and her own interests, but also to the imperialist way of thinking, to the liberalism of the rising middle classes and to the control society, embodied in the Panoptic, that already begins to consolidate in those times. Through the analysis of certain aspects of his gaze (not all), this article will try to better understand the different ideologies and social forces that acted on individuals during the second half of the 19th century.

Key words: Dracula, Panoptic, Liberalism, Imperialism, Mina Murray.

\section{LA "FICCIÓN"Y LA MIRADA}

Las ficciones, los mundos imaginados creados a lo largo de la historia, no surgen de la nada. Tampoco nacen única y exclusivamente de la imaginación de sus autores. Como ya apuntara Raymond Williams y recuerda Isabel Burdiel,

Data de recepció: 27 de maig de 2019 / Data d'acceptació: 6 d'octubre de 2019. 
los humanos somos seres sociales que trabajamos, comemos, miramos e imaginamos a la vez (Burdiel, 1996, 3). No podemos aislar determinados aspectos de hombres y mujeres y tratar tan sólo una parte de ellos. Hay que contemplar las múltiples facetas de su existencia, pues las unas influyen sobre las otras y conforman un todo; fraccionado y contradictorio, sí, pero un todo que debe ser interpretado, al que hay que darle un sentido de entre los múltiples posibles. ¿Realmente creemos que podemos estudiar a una sociedad sin tener en cuenta lo que esa sociedad fantasea sobre sí misma, sin reflexionar sobre lo que teme o desea, sin plantearnos cómo imagina que será tu futuro? Lo que finalmente no sucedió, lo imaginado, lo deseado y no acontecido, lo por largo tiempo temido y que posteriormente no se cumplió, nos aportan claves importantes que no podemos desechar, que como historiadores nunca deberíamos haber olvidado.

Cuando una persona idea un mundo, muchos de los elementos que lo constituyen provienen de la realidad extraliteraria o extraartística de su tiempo. Las terribles batallas que se suceden en un universo tan alejado del nuestro como el del Señor de los Anillos tienen su razón de ser en la experiencia de Tolkien durante la Primera Guerra Mundial. De este modo, aquella relación dialéctica entre pasado y presente expresada por Marc Bloch en su Introducción a la historia es igualmente aplicable a los vínculos que se establecen entre lo imaginado y lo real (aunque claro, lo imaginado también forma parte de lo real, es una parte de la realidad y tiene efectos sobre ella) (Bloch, 2001, 38-39).

En cualquier caso, y nos refiramos a estas nociones con los vocablos que sea, existe una relación dialéctica entre lo "imaginado" y lo "real" (aunque en verdad se trata de una relación triple, como enseguida veremos) $)^{1}$. Saber de la Primera Guerra Mundial puede permitirnos captar determinados matices presentes en la escritura de Tolkien; comprender mejor el terror que entre las tropas provocan los Nazgul montados a lomos de sus Bestias Aladas. Por otro lado, analizar la camaradería que se establece entre los diferentes ejércitos de El señor de los anillos en su enfrentamiento con un enemigo común, y el horror que todo ello provoca, puede ayudarnos a profundizar en nuestros conocimientos sobre las atrocidades sucedidas durante la Gran Guerra. A esta relación dialéctica se le añade, sin embargo, un tercer elemento: si captar lo vivo es una de las cualidades que debe tener todo buen historiador (Bloch, 2001, 38), resulta innegable que las ficciones de las que nos ocupamos, por muy antiguas en el tiempo, o lejanas en el espacio que nos parezcan, conservan un conoci-

${ }^{1}$ La dualidad "real" e "imaginario", es compleja y rica, y ha sido abordada por distintos estudiosos. Aunque no es objeto del presente artículo abordar en profundidad ambos conceptos, sino constatar que lo "imaginario" también forma parte de lo "real", remito al lector a los estudios de Todorov (2016), Roas (2011) y Carroll (2005). 
miento, un saber propiamente humano, del que podemos sacar provecho. En el caso de Tolkien, el horror de la guerra, el dolor, el sufrimiento y el sacrificio que provoca. El valor del respeto y de la paz. O cómo la tecnología (el anillo), puede acabar sometiendo y destruyendo a los hombres.

Toda ficción, por tanto, tratada con las técnicas y los procedimientos adecuados, está en condiciones de hacernos entender mejor a la sociedad que la produce (Lillo, 2017a, 267-288). De igual modo, entender la sociedad que la produce nos permite enriquecer nuestra propia lectura de la obra, ayudándonos a extraer así, aunque no lo parezca, lecciones de carácter más universal. Siguiendo estos mismos principios voy a explicar aquí la forma de mirar el paisaje de un personaje de ficción: Mina Murray, una de las protagonistas de Drácula, la novela publicada por Bram Stoker en 1897. A través del estudio de un aspecto de su mirada demostraré esa relación dialéctica existente entre lo "real" y lo "imaginario", y cómo gracias a este tipo de análisis puede obtenerse alguna enseñanza que nos permita entender mejor el mundo de hoy.

Conviene aclarar, sin embargo, que el análisis de la mirada de Mina va a ser parcial, y que va a atenerse tan sólo a algunas de sus características: las relacionadas con el pensamiento liberal en ascenso, la mirada imperial y la sociedad de control. Como ya se ha defendido en distintos lugares (Lillo, $2019 \mathrm{y}$ 2017b, 125-246), Mina no es sólo uno de los personajes más fascinantes y complejos de Drácula, sino que además despliega una mirada y una actitud decididamente feminista. Dejaremos de lado aquí, por consiguiente, tanto su mirada en pro de la igualdad entre los sexos como su relación con los varones que supuestamente la protegen (Jonathan Harker, Abraham van Helsing, John Seward), para centrarnos, tras una reflexión genérica sobre "el mirar", ...en su primer encuentro con Drácula. Entonces, profundizaremos en los mecanismos sociales que actúan sobre Mina y que le permiten oponerse a él.

\section{LUZ INTERIOR}

Mirar un paisaje, como afirma Eric Hobsbawm, tiene muchos elementos en común con mirar hacia una parte del pasado. "Mirar hacia atrás, o hacia adelante o en cualquier otra dirección siempre implica un punto de vista" (Hobsbawm, 2003, 17). Es un punto de vista que tiene que ver con el espacio que ocupa el observador y el tiempo (histórico) desde el que observa. Mirar, y da igual lo que miremos, implica también una selección. Y exige, aunque no lo parezca, un aprendizaje previo.

El sentido de la vista, como sucede con el olfato, el tacto, el gusto o el oído, es un mecanismo perceptivo que necesita ser educado. A mirar (como a oler o 
a escuchar) se aprende ${ }^{2}$ Y cada uno puede aprender a su manera. Debido a ese carácter formativo de los sentidos, no todos los individuos observan, escuchan o tocan igual; no todo el mundo, al contemplar un mismo objeto, aprecia idénticas cosas. En función de las cualidades de cada mirada se resaltarán unos rasgos y se ignorarán los restantes, se dirigirá la atención hacia determinados objetos o composiciones y se descartarán otros.

Los seres humanos hemos interiorizado tanto este proceso que consideramos el acto de ver más como un suceso natural, inherente a nuestra propia naturaleza, que como una habilidad asimilada (Cosgrove, 2002, 66). No somos conscientes de que, al abrir los ojos cada mañana, lo que apreciamos es "un mundo que hemos pasado toda una vida aprendiendo a ver", un mundo que vamos construyendo pieza a pieza a cada momento (Sacks, 2015, 152).

Lo cierto es que la visión necesita de varios elementos, aparte de la luz, para ser tal. No basta con disponer de un órgano visual que, mediante impulsos electroquímicos, transmita lo que capta del exterior hacia el cerebro. Si el interior del individuo está vacío, la luz del afuera no tendrá nada contra lo que reverberar.

Durante los primeros años de vida, existe un período crucial en el que se forman no sólo las capacidades visuales, sino muchas otras de carácter sensorial o motriz, como la de hablar o la de andar. Si la oportunidad no se aprovecha, recuperarla más adelante supone una dificultad enorme, y los resultados suelen ser infructuosos (Zajonc, 2015, 17).

Es lo que sucede con ciertas personas ciegas desde la infancia que, tras alguna operación, recuperan la vista. Según revelan diferentes estudios científicos, aprender a ver, en el caso de los adultos, es una tarea muy ardua y desasosegante. En algunos de estos casos los pacientes incluso necesitan tocar previamente los objetos para reconocerlos. Sólo entonces están en disposición de identificarlos con la vista. Es lo que sucedió con un muchacho de trece años, ciego de nacimiento, que fue operado en 1728 por William Cheselden. Este joven, que "no tenía ni idea de la distancia", ni del "espacio ni del tamaño", únicamente "comprendía lo que veía poco a poco, y en la medida en que era capaz de relacionar las experiencias visuales y las táctiles" (Sacks, 2015, 147148). También fue el caso de S. B., un varón de cincuenta años ciego desde los diez meses de edad que, tras una operación de córnea, recuperó la visión en

2 Aunque "mirar" y "ver" no son palabras sinónimas, en el texto que sigue se emplearán en un sentido similar. Sobre las diferencias entre ambas expresiones, ver Moreno de Alba (1996, 375-376) y Sacks $(2015,155-156)$. 
ambos ojos. De visita al Museo de Ciencias de South Kensington (actualmente el Museo de Ciencias de Londres), tuvo que palpar un torno que mecanizaba tornillos para poder verlo ${ }^{3}$. Lo que este tipo de estudios ponen de manifiesto, aparte de generar controversias que llegan hasta nuestros días, es el impresionante reto al que se enfrenta la comunidad científica debido a la dificultad de entender a fondo los mecanismos de la percepción.

Así pues, mirar es un ejercicio complejo que pone en juego muchas de nuestras capacidades, incluso el resto de los sentidos. Pese a que generalmente la visión se estudia de forma aislada, el gusto, el sonido y otras experiencias sensoriales, dotan de riqueza y nuevos significados a las imágenes de la retina (Gregory, 2004, 836). No miramos sólo con los ojos, sino con todo nuestro ser, con todo lo que somos.

Por ese motivo para ver no basta con un ojo sano y con la luz del exterior. Es necesaria también una "luz interior" que transforme lo que percibimos y le confiera un sentido: "La luz de la mente tiene que fluir y unirse con la luz de la naturaleza para crear el mundo" (Zajonc, 2015, 18). Los seres humanos necesitamos de toda nuestra inteligencia y facultades, de nuestro particular bagaje cultural, para dotar de significado a lo que vemos. El hecho de mirar se convierte así en un acto cultural; un acto cultural que no sólo varía de una persona a otra, sino que se modifica con el paso del tiempo. Cada sociedad tiene formas propias de ver el mundo y, por tanto, también de representarlo (Panofsky, 2003, 27).

\section{DISTANCIA, CONOCIMIENTO Y PODER}

Una vez aclarado este extremo se hace necesario resaltar que Mina tiene una mirada muy particular. Durante sus vacaciones de verano, que pasa en la costera localidad de Whitby, y mientras su prometido Jonathan Harker está en el castillo de Drácula, la joven lleva un diario. Mina decide realizar parte de sus anotaciones en un banco situado en lo alto de un cementerio, el punto más hermoso del lugar. Desde allí la joven no sólo disfruta de una vista magnífica, también adquiere un panorama completo del puerto y la bahía, de la ciudad y sus alrededores. Desde ese banco Mina realizará la mayoría de sus anotaciones sobre el paisaje. El hecho de que decida escribir su diario desde lo alto de una colina, observando el paisaje, con la hermosa vista del horizonte y la inmensidad del mar, nos habla de la amplitud que Mina quiere darle a su mirada:

${ }^{3}$ Un informe sobre este caso, estudiado en 1963 por Richard Gregory y Jean G. Wallace, puede encontrarse en: http://www.richardgregory.org/papers/recovery_blind/4-obsdischarge.htm. (Consultado el 06-04-2018). Ver también Sacks (2015, 173-174). 
Un pequeño río, el Esk, discurre a través de un profundo valle, que se ensancha a medida que se acerca al puerto. Lo atraviesa un gran viaducto (...). Todas las casas de la ciudad antigua (...) tienen los tejados rojos y parecen haber sido apiladas una sobre la otra de cualquier manera (...). Justo sobre la ciudad se alzan las ruinas de la abadía de Whitby (...). Entre la abadía y la ciudad hay otra iglesia, la parroquial, rodeada de un gran cementerio lleno de tumbas (...). A mis pies se extiende el puerto, en cuyo extremo más alejado un largo muro de granito se interna en el mar, acabando en una curva en mitad de la cual hay un faro... (Stoker, 2010, 147-148).

El río, un profundo valle y un enorme viaducto, por un lado; las casas de la ciudad antigua por otro; sobre el pueblo, las ruinas de una abadía, la iglesia parroquial y un enorme cementerio; algo más allá, el puerto, un largo muro de granito que se interna en el mar y el faro. El cuadro que dibuja Mina es claro y preciso, está perfectamente descrito y ordenado. Encaramada a un promontorio, Mina obtiene una visión general de todo cuanto la rodea.

Ubicada en una posición estratégica, se aleja del mundo en el que está viviendo y pasa a describir una escena que contempla (Cosgrove, 2002, 71 y Wylie, 2007, 7). El entorno se convierte así en un escenario distante que puede ser observado y representado como algo ajeno. Sin embargo, como he tratado de argumentar un poco más arriba, el paisaje que describe Mina no sólo está ahí fuera; también se encuentra en los ojos que miran. Permítaseme recordarlo: la interacción de ambos elementos, así como lo que anida en el interior de la joven, da como resultado una construcción, la composición de un mundo. Esa mirada, que podríamos llamar paisajística, no es más que una de las formas de ver la realidad en la que ha sido educada. Al fin y al cabo, el gusto por el paisaje en Inglaterra fue consecuencia directa del Gran Tour, convirtiéndose, ya desde comienzos del siglo XIX, en un rasgo cultural de las clases medias (Hussey, 2013, 38 y Cosgrove, 2002, 74). Mina emplea sus gustos pictóricos, fotográficos y literarios para dar forma y ordenar lo que ven sus ojos. Pero lo más importante es que se vale de la distancia, de ese alejarse de lo que observa, para poder distinguir con claridad. Como afirma Franco Moretti, "la distancia, ciertamente, permite apreciar menos detalles, pero ayuda a comprender mejor las relaciones, los patterns, las formas" (Moretti, 2007, 10). Al elegir ese promontorio como lugar de observación, Mina opta por la distancia como forma de conocimiento. Nos indica, además, que su mente está abierta, dispuesta a mirar con la mayor amplitud posible los sucesos o los acontecimientos que percibe. La joven tiene la voluntad de captar al completo lo que puede apreciar con sus sentidos. Para ello no sólo empleará los ojos; también los oídos y el olfato, su bagaje cultural y sus prejuicios, todo lo que ella es: 
Veo luces desperdigadas por toda la ciudad, a veces en hileras, siguiendo el recorrido de las calles, otras veces solitarias; bordean toda la ribera del Esk hasta morir en la curvatura del valle. A mi izquierda, la vista se ve interrumpida por la negra silueta del tejado de la vieja casa que hay junto a la abadía. Las ovejas y los corderos balan en los campos, lejos, a mis espaldas, y puedo oír el ruido de los cascos de un burro ascendiendo por la pavimentada carretera de abajo. La banda está tocando un estridente vals a buen ritmo en el malecón, y más avanzado el muelle hay una reunión del Ejército de Salvación, en una calle lateral. Ninguna de las bandas puede oír a la otra, pero desde aquí arriba las oigo y las veo a ambas (Stoker, 2010, 155).

Como puede apreciarse, su mirada es ambiciosa e integradora. Mina emplea todos sus recursos, incluida la imaginación, para "mirar", para descubrir un paisaje y trasladarlo al papel de su diario. La muchacha describe la disposición exacta de las luces; también los límites de su visión, esa "negra silueta" de una casa que identifica perfectamente y que puede distinguirse en la distancia pegada a la abadía. Percibe además lo que está sucediendo en puntos concretos que no puede ver: identifica a las ovejas por los balidos que oye a sus espaldas o el sonido de las herraduras de un asno al avanzar por la carretera.

Desde su posición parece verlo y controlarlo todo. Sin embargo, pese a la meticulosidad que desprende su texto, la clave del fragmento se encuentra en el comentario que realiza sobre las dos bandas de música: una de ellas está tocando en el malecón; la otra interpreta una sinfonía algo más lejos, en una calle lateral. Una vez detallada la ubicación de los grupos es cuando añade: "Ninguna de las bandas puede oír a la otra, pero desde aquí arriba las oigo y las veo a ambas". Esta frase es fundamental y confirma lo dicho hasta ahora de la joven. Mina ve lo que otros, más implicados en los hechos que ella describe o analiza, no pueden percibir o son incapaces de descubrir: ninguna de las bandas puede oír a la otra, pero gracias a su ubicación ella las ve y las oye a ambas. Gracias a la posición distanciada que adopta y a las peculiaridades de su mirar, lo que ella percibe y transmite se convierte en información privilegiada: la mirada de Mina también es poder.

Si el éxito de Drácula depende de su capacidad para pasar inadvertido en un entorno tan populoso como Londres y sus alrededores, el éxito del grupo que lo persigue depende en gran medida de Mina, de la capacidad que tiene para, desplegando todas sus cualidades e inteligencia, observar el problema desde la distancia y encontrar la solución. Si el poder de Mina está en su mirada, las aspiraciones de Drácula descansan en la ocultación, en no ser descubierto antes de tiempo.

Esa lucha de la una por ver y del otro por no ser visto se libra por primera vez en Whitby. Allí asistimos a un duelo de dimensiones épicas. Drácula acaba 
de desembarcar en la localidad inglesa transformado supuestamente en un enorme perro. Desde ese momento permanece prácticamente oculto: su presencia sólo puede ser intuida por el lector del diario de Mina en unos pocos pasajes. Sin embargo, al amparo de la tranquilidad y el sosiego que proporciona la noche, el vampiro parece estar mordiendo a Lucy, e incluso en una ocasión, mientras la muchacha se encuentra en estado sonámbulo, la atrae hasta uno de los bancos del cementerio. Cuando Mina descubre la escapada nocturna de su amiga, sale en su busca ansiosa por saber, nerviosa por descubrir su paradero. Espera distinguir una figura vestida apenas con un camisón blanco. Desde ese momento se aprecia la importancia que en dicho episodio van a desempeñar las palabras relativas a la visión, a la dificultad de ver, que es una de las formas del conocer:

El reloj daba la una cuando salí a la calle Crescent y no se veía ni un alma (...). No pude ver ni rastro de la blanca silueta que estaba buscando (...). Había un claro de luna brillante sobre el que flotaban unas nubes negras y pesadas. (Stoker, 2010, 189)

Ya se ha comprobado lo inquisitiva que puede llegar a ser la mirada de Mina; también lo evasivo que se muestra Drácula. Es así como, escindidos entre la brillante claridad de la luna ("a bright full moon") y las pesadas nubes negras ("heavy black, driving clouds"), los lectores asistimos a un juego de ocultación y discernimiento ${ }^{4}$. En un principio las nubes impiden ver el panorama, pero al pasar el nubarrón Mina distingue, gracias a la luz de la luna, la blanca figura que está buscando. Sin embargo, casi en ese mismo instante las sombras vuelven a inundarlo todo. Ese rápido vistazo, empero, es suficiente para Mina: ha logrado distinguir su objetivo.

Pero me pareció ver como si una forma oscura se alzara desde detrás del banco en el que había resplandecido la blanca figura, inclinándose sobre ella. Pero no podría haber asegurado si se trataba de hombre o bestia. (Stoker, 2010, 189)

Aunque la impresión es fugaz, Mina percibe, sin espacio para la duda, una forma oscura, quizá de origen bestial, que se contrapone a la blancura que

\footnotetext{
4 "Durante unos momentos fui incapaz de ver nada, ya que la sombra de una nube ocultaba la iglesia de St. Mary y todo lo que la rodeaba. Entonces, cuando la nube terminó de pasar, vi aparecer las ruinas de la abadía (...); sobre nuestro banco favorito, la plateada luz de la luna iluminó una figura medio reclinada, de un blanco níveo. El paso de la nube fue demasiado rápido como para permitirme ver demasiado, pues las sombras ahogaron la luz casi de inmediato" (Stoker, 2010, 189).
} 
emana de Lucy. En este punto, la alegoría de la escena se multiplica exponencialmente: a la lucha por ver y no ser visto, transformada ya en una especie de duelo entre la luz y la oscuridad (la luz de la luna y la blancura de Lucy frente a la negrura de las nubes y la oscuridad de la misteriosa figura), se le añade un nuevo elemento simbólico. Si Lucy representa la belleza y la inocencia, ese ser tenebroso que se inclina sobre ella encarna una especie de monstruosidad malvada. De este modo, bondad, belleza, luz y conocimiento quedan vinculados en la descripción de Mina. Y frente a ellos, la maldad, la oscuridad, la monstruosidad y la ignorancia, representada por el vampiro.

Este duelo entre la luz y la oscuridad, encarnado respectivamente en Lucy y en Drácula, puede entenderse con facilidad como una manifestación más de la eterna lucha del bien contra el mal, de las aspiraciones del demonio para corromper y contagiar a los ángeles. Es algo que también puede apreciarse nítidamente en el diario de Jonathan Harker ${ }^{5}$.

Sin embargo, si nos atenemos a ciertas características de la mirada de Mina, podríamos superponer a esa lucha milenaria un nuevo elemento que quizá enriquecería la visión del conjunto. Si convenimos en que la mirada de Mina está formada por un elemento personal y otro social, vinculado con una forma culturalmente determinada de "ver", estaríamos en condiciones de argumentar que esa ubicación privilegiada desde la que observa, así como su voluntad de describir con precisión milimétrica la totalidad del entorno, sin perder detalle alguno y sin ser vista, recuerda al Panóptico, un ingenio de la vigilancia ideado por Jeremy Bentham en 1787.

El Panóptico es un edificio que tiene como principal función el control de un amplio conjunto de personas con el menor esfuerzo y coste posible. El edificio adopta una forma circular, con dos espacios claramente delimitados. En la circunferencia exterior se encuentran las celdas de los prisioneros. En el centro de la circunferencia se erige una torre. Así, un único centinela puede vigilar todas las celdas sin moverse de su puesto; igualmente, gracias a la disposición arquitectónica y a determinados juegos de luces, los presos nunca estarán en condiciones de ver al vigilante. No tienen forma de saber si se les está controlando o no.

Aunque la mirada de Mina no es cien por cien panóptica (entre otras cosas porque el Panóptico es una estructura que debe construirse en un espacio cerrado y poseer unas características muy determinadas), sí contiene elementos que recuerdan a ese invento. El Panóptico, al fin y al cabo, "permite que exista un lugar desde el que, sin cambiar de situación, se pueda contemplar de manera

5 Ver, por ejemplo, la actitud de Drácula ante las mujeres vampiro, en Stoker, 2010, 111-112. 
cabal todo el conjunto al mismo tiempo" (Bentham, 2011, 59). La referencia a las dos bandas de música que tiene controladas Mina pero que no pueden verse entre ellas vuelve a ser significativa. Así lo expresa Michel Foucault refiriéndose al Panóptico de Bentham: "Cada cual, en su lugar, está bien encerrado en una celda en la que es visto de frente por el vigilante; pero los muros laterales le impiden entrar en contacto con sus compañeros. Es visto, pero él no ve; objeto de una información, jamás sujeto en una comunicación" (Foucault, 2009, 204). En el caso que nos ocupa, son las calles laterales las que actúan como muros que impiden que las bandas puedan verse, e incluso oírse. Mina, por su parte, permanece en su atalaya tratando de controlarlo todo, transmitiendo a través de su mirada gran cantidad de información y ejercitando una forma de ver que resultará muy útil en su lucha contra Drácula.

Pero las similitudes no acaban ahí. El poder del Panóptico descansa en varios elementos. Uno de ellos, que resulta fundamental, es el de la iluminación. La disposición de las celdas se realiza de tal manera que la luz inunda el espacio del Panóptico. El resultado es que ninguna parte de la celda queda oculta a la mirada del vigilante. La luz, como escribe Jonathan Crary, debe inundar el espacio del Panóptico "para eliminar las sombras y hacer de la condición de plena observabilidad un sinónimo del efecto de control" (Crary, 2015, 26). Es eliminando la oscuridad y haciéndolo todo visible, como comienzan a funcionar en el Panóptico los mecanismos de poder que aspiran a una vigilancia total y absoluta sobre los individuos. "La plena luz y la mirada de un vigilante captan mejor que la sombra, que en último término protegía. La visibilidad es una trampa" (Foucault, 2009, 204).

Situados en estas coordenadas, el duelo entre luz y oscuridad, entre visibilidad y ocultación al que asistimos en Whitby, adquiere un nuevo sentido. La mirada de Mina encarna esa sociedad de la vigilancia sobre la que teorizara Foucault, una mirada que prolonga los "efectos de poder" provocados por la "máquina panóptica", esa que "lo domina todo de una sola mirada" (Foucault, 2009, 220); esa en la que la visibilidad se convierte en el primer paso para el sometimiento.

Drácula, al fin y al cabo, viene de un lugar que no está en los mapas del Museo Británico, o al menos así lo afirma Jonathan: "No fui capaz de encontrar ningún mapa o tratado que iluminara la localización exacta del castillo de los Drácula" (Stoker, 2010, 58). Vuelve a ser la luz la clave del desvelamiento, aspecto este último que no es más que el paso previo del dominio y del control. Era así, mediante la iluminación o el descubrimiento, como el imperialismo europeo iniciaba su proceso de apropiación: 
El descubrimiento era el acto de nombrar y ver -por un hombre blanco- lo que otros ya conocían; esto es, en la práctica, desplazarse hasta el lugar en cuestión, averiguar por los nativos dónde había un gran lago o un gran río, luego contratarlo [sic] para que el explorador fuera conducido hasta el sitio y por último bautizar el lugar con un nombre propio europeo (por ejemplo, el Lago Victoria) para reivindicar a continuación los derechos territoriales nacionales sobre dicha geografía. La violencia simbólica del descubrimiento es el prolegómeno a la implantación de los colonos y el posterior reclamo de la propiedad y usufructo privado de la tierra y sus riquezas (Gasquet, 2006, 40).

Sin mirada, sin iluminación, no hay dominio. El vampiro y su fortaleza están fuera del alcance del ojo imperial, ese proyecto de exploración y conquista que comienza en el siglo XVIII y que a finales del XIX ha desplegado todo su poderío (Pratt, 2010). Desde una posición privilegiada -la metrópolise puede divisar y controlar el todo; nada queda fuera de su mirada. Dicho proceso de colonización e imperialismo revela una obsesión por descubrir -por ver o iluminar- todas las regiones del orbe que hasta entonces permanecían inexploradas, y rellenar así, en el mapa del mundo, los espacios que permanecen en blanco (Youngs, 2006). ¿El objetivo? Cartografiarlos, catalogarlos y, finalmente, controlarlos para someter a sus habitantes. El castillo de Drácula escapa a esa mirada imperial de igual manera que el boyardo se escabulle como puede de la de Mina. La mirada de la joven, en este sentido, también es imperialista, también busca visibilizar a la amenaza extranjera para desactivarla, no dejándole ni un espacio de sombra en donde protegerse.

Mina está controlando a Lucy, preocupada por su extraña enfermedad, pero también controla todo cuanto sucede en Whitby. Está vigilante sin saberlo, atenta a una posible invasión en lo que sería un proceso de colonización inversa ${ }^{6}$; al fin y al cabo se encuentra en una localidad costera que ya ha sido saqueada por los daneses (Stoker, 2010, 147). Podría suceder que los bárbaros volvieran a intentarlo. ¿Acaso Drácula no tiene sangre vikinga? Así se lo comenta el vampiro a Jonathan: "Nosotros los szekler nos hemos ganado el derecho a ser orgullosos, pues por nuestras venas fluye la sangre de muchas razas valientes" (Stoker, 2010, 97). Una de ellas lo emparenta con los reinos vikingos nórdicos -también daneses-, que "con feroz intensidad desplegaron sus bersekers en las costas de Europa" (Stoker, 2010, 97).

${ }^{6}$ El artículo de referencia sobre la colonización inversa en Drácula es el de Arata (1990, 621-645). Sin embargo, el primer texto que apunta en esa línea posiblemente sea el de Brantlinger (1985, 243-252). 
De este modo Mina se erige en guardiana, no sólo ante un proceso de conquista que emularía lo que los propios británicos estaban haciendo en distintas partes del mundo, sino también de los adelantos de su civilización frente a la barbarie del exterior. Drácula, al fin y al cabo, es un señor feudal. De este modo, el duelo entre la iluminación y las sombras que representan Mina y Drácula reproduciría los esfuerzos de la democracia liberal por acabar con el oscurantismo del Antiguo Régimen. El vampiro es un noble que basa su dominio en unos privilegios que le vienen por nacimiento. El poder que ejerce sobre su tierra es el de las sombras y el ocultamiento, el de la mazmorra, la opacidad y la arbitrariedad, y es eso lo que quiere llevar consigo a Inglaterra. Desde la segunda mitad del siglo XVIII los esfuerzos de los revolucionarios burgueses han ido encaminados a conseguir todo lo contrario. "Disolver los fragmentos de noche que se oponen a la luz, hacer que no existan más espacios oscuros en la sociedad, demoler esas cámaras negras en las que se fomenta la arbitrariedad política, los caprichos del monarca, las supersticiones religiosas, los complots de los tiranos" (Foucault, 1979, 16).

La mirada que encarna Mina, o al menos una parte de esa mirada (la del liberalismo de las clases medias en ascenso), también aspira a crear "una sociedad transparente, visible y legible", una sociedad en la que "no existan zonas oscuras, zonas ordenadas por los privilegios del poder real o por las prerrogativas de tal o cual cuerpo" (Foucault, 1979, 15). La mirada de Mina, en ese sentido, es contraria a la tiranía que podría representar Drácula. Sin embargo, pronto es víctima de su propia obsesión por la transparencia, por su ineluctable deseo de mostrarlo todo, como si no fuera necesario un espacio de sombra desde el que relajarse y reflexionar, desde el que tratar de entender mejor al otro sin tener que acarrear con lo que cada uno es en cada momento. Mina debería saberlo, sometida como está a un control constante por parte de casi todas las instancias morales que imperan en su sociedad, pero la carga social de su mirada es más fuerte que la de su propia experiencia. Así es como la joven se comporta, sin pretenderlo, como una oprimida que oprime. Se convierte en una mujer que, condenada como está a cambiar constantemente de máscara para sobrevivir, obliga al otro, al diferente, a no desprenderse en ningún momento de la suya, a no disponer de ningún espacio de relax en el que mostrarse sin la presión de ser alguien, dificultando así cualquier posibilidad de acuerdo o diálogo. La mirada de Mina, una parte de esa mirada, en la medida en que colabora con los varones, no le deja alternativa a Drácula.

La mirada de Mina, siendo extraordinaria, no escapa a los dictados de su tiempo. Está hecha de retazos y no puede desprenderse ni de lo que ella es, ni de la tradición de la que depende, ni de la sociedad en la que vive. Se trata de un mirar complejo en el que se mezclan y superponen distintas formas de ver 
el mundo. A la analítica y racional se suma esa visión controladora y totalizadora característica del Panóptico; a la mirada imperialista, esa que trata de negar al otro, al diferente, se solapa otra más inquisitiva que aspira, heredera de la mejor tradición del periodismo, al conocimiento y la verdad. Cada una de esas formas de ver tiene sus raíces, su historia y sus desarrollos y, en tanto pugnan por imponerse unas sobre las otras, no cristalizan en Mina exentas de conflictos y contradicciones. En los ojos de Mina y en el modo que tiene de construir la realidad son distintas las formas de ver que pugnan por imponerse.

Al tiempo que contribuye decisivamente a acabar con Drácula, la mirada humana de Mina siente lástima por él: "Supongo que una debería compadecerse de una criatura tan acosada como el Conde" (Stoker, 2010, 392). Pese a todos sus condicionantes sociales, la joven vislumbra en Drácula a un igual, alguien con el que identificarse. Quizá el noble transilvano no sea ese ser monstruoso y horrible que nos describe Jonathan Harker en su diario. Quizá el acoso al que es sometido no le deje otra alternativa que comportarse como se comporta; quizá se ve obligado a colocarse una máscara de la que ya está harto para poder sobrevivir en un entorno inhóspito y extraño. A falta de espejos quizá es la mirada del otro la que lo transforma en un ser monstruoso. Pero antes siquiera de pensar esto hay que estar en disposición de verlo.

\section{BIBLIOGRAFÍA}

ARATA, S. D. (1990): "The Occidental Tourist: Dracula and the Anxiety of Reverse Colonization", Victorian Studies, 33 (Summer), 621-645.

BENTHAM, J. (2011): Panóptico, Madrid, Círculo de Bellas Artes, 153 p.

BLOCH, M. (2001): Introducción a la historia, Madrid, Fondo de Cultura Económica, $159 \mathrm{p}$.

BRANTLINGER, P. (1985): "Imperial Gothic: Atavism and the Occult in the British Adventure Novel, 1880-1914”, English Literature in Transition, 1880-1920, 28-3, 243-252.

BURDIEL, I (1996): "Lo imaginado como materia interpretativa para la historia", en: Burdiel, I. y Serna, J., Literatura e historia cultural. Por qué los historiadores deberíamos leer novelas, Valencia, Episteme, 1-22.

CARROLL, N. (2005): Filosofía del terror o paradojas del corazón, Madrid, Machado Libros, $488 \mathrm{p}$.

COSGROVE, D (2002): "Observando la naturaleza: el paisaje y el sentido europeo de la vista", Boletín de la Asociación de Geógrafos Españoles, 34, 63-89.

CRARY, J. (2015): 24/7. El capitalismo al asalto del sueño, Barcelona, Ariel, 144 p. FOUCAULT, M. (1989): “«El ojo del poder», entrevista con Michel Foucault”, en: Bentham, J., El Panóptico, Barcelona, La Piqueta, 9-26. 
FOUCAULT, M. (2009): Vigilar y castigar. El nacimiento de la prisión, Madrid, Siglo XXI, $314 \mathrm{p}$.

GASQUET, A. (2006): “«Bajo el cielo protector». Hacia una sociología de la literatura de viajes", en: Lucena Giraldo, J. y Pimentel, J. [eds.], Diez estudios sobre literatura de viajes, Madrid, CESIC - Consejo Superior de Investigaciones Científicas, 31-66.

GREGORY, R. L. (2004): “The blind leading the sighted”, Nature, 430, 836.

HOBSBAWM, E. (2003): Los ecos de la marsellesa, Barcelona, Crítica, 174 p.

HUSSEY, CH. (2013): Lo pintoresco. Estudios desde un punto de vista, Madrid, Biblioteca Nueva, 384 p.

LILLO, A. (2017a): "La literatura de ficción como fuente histórica”, Studia Historica. Historia Contemporánea, 35, 267-288.

LILLO, A (2017b): Miedo y deseo. Historia cultural de Drácula (1897), Madrid, Siglo XXI, $366 \mathrm{p}$.

LILLO, A. (2019): "La construcción de la identidad en Drácula", en: Moreno Seco, M. [coord..], Del siglo XIX al XXI. Tendencias y debates: XIV Congreso de la Asociación de Historia Contemporánea, Alicante, Biblioteca Virtual Miguel de Cervantes, $2019 \mathrm{p}$.

MORENO DE ALBA, J. G. (1996): Nuevas minucias del lenguaje, México, Fondo de Cultura Económica, 435 p.

MORETTI, F. (2007): La literatura vista desde lejos, Barcelona, Marbot Ediciones, $159 \mathrm{p}$.

PANOFSKY, E. (2003): La perspectiva como forma simbólica, Barcelona, Tusquets, $176 \mathrm{p}$.

PRATT, M.L. (2010): Ojos imperiales. Literatura de viajes y transculturación, México D.F., Fondo de Cultura Económica, 471 p.

ROAS, D. (2011): Tras los límites de lo real, Madrid, Páginas de Espuma, 192 p.

SACKS, O. (2015): "Ver y no ver", en: Un antropólogo en Marte, Barcelona, Anagrama, 145-194.

STOKER, B. (2010): Drácula, Madrid, Valdemar, 681 p.

TODOROV, T. (2016): Introducción a la literatura fantástica, Ciudad de México, Coyoacán, $188 \mathrm{p}$.

YOUNGS, T. [ed.] (2006): Travel Writing in the Nineteenth Century, London, New York, Anthem Press, $268 \mathrm{p}$.

WYLIE, J. (2007): Landscape, London and New York, Routledge, 246 p.

ZAJONC, A. (2015): Capturar la luz, Girona, Atalanta, 387 p. 